\title{
Isocyanide-based multicomponent reactions in the synthesis of heterocycles
}

\author{
Majid M. Heravi ${ }^{1}$, Samahe Sadjadi, Niousha Nazari \\ ${ }^{1}$ Department of Chemistry, Faculty of Physics \&Chemistry Alzahra University, Vanak, Tehran, Iran.
}

\begin{abstract}
In this review, we update our previous presentation, underscoring the recent applications of isocyanides as privileged synthons in the synthesis of various heterocyclic compounds, especially focused on those synthesized via multicomponent reactions.
\end{abstract}

\section{Keyword}

Cyanides, Heterocycles, Heterocyclic compound, Isocyanides, Multi-component reactions, Synthons, Synthesis (chemical) 